\title{
THE USE OF GAS PROPORTIONAL COUNTERS TO DISTINGUISH PROTONS FROM PIONS IN THE COSMIC RADIATION AT ENERGIES OF NEAR OR GREATER THAN 100 GeV*
}

\author{
P V RAMANA MURTHY ${ }^{+}$and G D DEMEESTER \\ Department of Physics, Unversity of Michigan, Ann Arbor, Michigan, US A
}

Recelved 19 June 1967

Several possible methods to distinguish protons and pions in the cosmic radiation at energies of over $100 \mathrm{GeV}$ are discussed One of these methods based on multiple sampling of ionization losses in an array of gas proportional counters together with a measurement of energy in an ionization calorimeter or momentum in a magnet spectrograph is discussed in greater detail

Fluctuatıons in ionızation losses ("Landau" fluctuations) make the method difficult but not impossible Experimental results on Landau fluctuations with a variety of incident particles

\section{Introduction}

A considerable amount of interest has arisen in the recent past in the possibility of utılızing high energy nuclear-active particles in the cosmic radiation to study high energy physics in the energy range $\gtrsim 100 \mathrm{GeV}$ with a precision comparable to the one in the experiments with machine produced particles This precision being much better than the one hitherto achieved in the traditional cosmıc ray experıments, one begins to think in more ambitious terms, e $g$, large extents of magnetıc fields, using for example superconductıng magnets and delineation of trajectories with a precision better than $01 \mathrm{~mm}$ using a multitude of spark chamber and nuclear emulsions over a large area While the emphasis is on precision, one has also to identify each incident particle High energy nuclear-actıve particles in the cosmic radiation consist of protons, neutrons, prons and, to a lesser extent ${ }^{\dagger}$, of kaons Identification of neutrons is trivial since they are the only long-lived neutral particles Negatıvely charged partıcles identıfied by the sense of curvature of their deflection in a known magnetic field can be labelled as negative pions Separation of protons and positive pions at these high energies $(\gtrsim 100 \mathrm{GeV})$ poses a real problem We shall mention the various methods one could use to attain this objective in section 2 and discuss the potentialities of what we consider the most promising in some detail. In section 3 , we shall present the behavior of the apparatus constructed to pursue the problem experimentally and discuss the practical implıcations Finally, a summary of the paper will be presented in section 4 .

\section{Methods}

Since both the pions and protons are electrically and energies are presented and it is shown that they are in better agreement with the theory of Blunck and Leisegang than with that of Landau

Artificial events in which the sampled ionization losses obeyed the Landau and Blunck and Leisegang distrıbutıons are generated on a computer by a Monte Carlo program Based on an analysis of a sample of 20000 such events by likelthood ratio method the attainable proton-pion separation in the cosmic radiation at 100 GeV energy is presented

charged, measurement of their deflection in magnetıc fields determines the momentum, an ionization calorimeter determines the total energy It might appear that a knowledge of momentum and energy enables one to determine the mass of the particle, but this is not so for $U \gg m c^{2}$ because the uncertanties in the measurements would prevent a meanıngful estımate of the mass to be made We mention this here only to rule it out of further discussion

In section 21 below, we shall consider the applicatıon of threshold gas Črenkov counters, a technique which was already successfully employed by Lal et al ${ }^{1}$ ) to distinguish pions and protons in the cosmic radiation in the energy range 7 to $40 \mathrm{GeV}$ Next, we discuss in section 22 the methods based on the relativistic rise of energy loss

\section{Threshold C̆erenkov COUNTERS}

Črenkov radiation is emitted by a charged particle only when its Lorentz factor, $\gamma\left[=U /\left(m c^{2}\right)\right]$, exceeds a certain value $\gamma_{t^{\prime}}$ which is dependent on the refractive index of the medium Recording the "yes" or "no" type of information from the Čerenkov counters and measurement of energy by a calorimeter or of momentum

* Research supported by the National Science Foundation and the U S Office of Naval Research Contract Nonr 1224(23)

+ On leave of absence from Tata Institute of Fundamental Research, Bombay-5, India

$\dagger$ Considerations of the $K / \pi$ ratio at production, life times and masses of kaons and pions and the $\tau+/ \mathrm{p}$ ratio in the atmosphere suggest that charged kaon flux in the atmosphere is of the order of $3 \%$ of the proton flux at energies $\approx 100 \mathrm{GeV}$ With the experımental techniques avallable today, it seems impractical to try to distinguish kaons from either protons or pions at these energies It appears therefore, that for the time being one must be willing to accept a proton or a pion beam whose purity cannot be known to better than $3 \%$ 
by deflecting in a magnetic field will enable one to distinguish the pions and protons in the energy range $m_{\pi} \gamma_{\mathrm{th}}<U<m_{\mathrm{p}} \gamma_{\mathrm{th}}$ where $m_{\pi}$ and $m_{\mathrm{p}}$ are the rest masses of pion and proton respectively. The refractive Index, $n$, of the medium and $\gamma_{\mathrm{th}}$ are related as

$$
n=\gamma_{\mathrm{th}} /\left(\gamma_{\mathrm{th}}^{2}-1\right)^{\frac{1}{2}}
$$

Since we are considering energies $>100 \mathrm{GeV}$, the high values of threshold Lorentz factors $\left(\gamma_{\mathrm{th}}>100\right)$ imply

$$
n=1+\delta,
$$

where $\delta$ is a very very small quantity indeed This, then allows the usage of gases only as the media for Cerenkov radiation $\delta$ and $\gamma_{\text {th }}$ are related by

$$
\gamma_{\mathrm{hh}}=(2 \delta)^{-\frac{1}{2}}
$$

The Cerenkov intensity integrated over the visible spectrum is given by [for example, ref $\left.{ }^{2}\right)$ ]

$$
I=500\left\{1-\left(\beta^{2} n^{2}\right)^{-1}\right\} \text { photons } / \mathrm{cm},
$$

which in terms of $\gamma$ and $\delta$ is given by

$$
I=500\left(2 \delta-\gamma^{-2}\right) \text { photons } / \mathrm{cm}
$$

The intensity of the Čerenkov radiation is zero just at the threshold $\left[\gamma=\gamma_{\mathrm{th}}=(2 \delta)^{-\frac{1}{2}}\right]$ and rises gradually to an asymptotic value of $1000 \delta\left(=500 / \gamma_{\text {th }}^{2}\right)$ photons $/ \mathrm{cm}$ at extremely high energies where $\gamma \gg \gamma_{\text {th }}$

Let us consider a specific example, namely, distınguishing pions and protons at energies around 100 $\mathrm{GeV}$, say in the energy range 70 to $140 \mathrm{GeV}$ The refractıve index of the gaseous medium in the Cerenkov counter has to be adjusted such that $\gamma_{\mathrm{th}}=150$ [or $\left.\delta=1 /\left(2 \gamma_{\mathrm{th}}^{2}\right)=225 \times 10^{-5}\right]$ in order that protons may not give a signal but only the pions do* From formula (5), the intensity of Cerenkov radiation emitted by pions of energy 70 to $140 \mathrm{GeV} \approx 21 \times 10^{-2}$ photons/ $\mathrm{cm}$ This figure is quite small and cannot be improved upon by playing with the nature of the gas, pressure, etc since it is essentially determined by the refractive index which in turn is determined by $\gamma_{\text {th }}$, which is fixed If the height of the Črenkov counter is $h \mathrm{~m}$, efficiency of light collection at the photocathode of the photomultiplier, $f$, and the quantum efficiency of the photocathode, $g$, the number of photo-electrons released at the photocathode is given by $21 \mathrm{fgh}$ In order not to lose much of the pion flux, $21 \mathrm{fgh}$ must at least equal 2 (Even in this case, one loses $\mathrm{e}^{-2}=135^{\circ}, 0$ of the pion flux) With a rather optimistic value of 02 for the product $f g$, one arrives at the value $h=48 \mathrm{~m}$ With these design parameters, one must recognize the existence of the following difficulties

1 While a signal from the Črenkov counter sig- nifies that the particle is a pion, its absence does not necessarily imply a proton, for the pions are counted only with a partial efficiency Thus a proton is never identıfied as such

2 The problem of recognızıng the signal from only two photoelectrons in the photomultiplier above the noise level requires special techniques

3 The rather large dimensions in the vertical scale (besides the necessarily required large horizontal dimensions) make the Cerenkov counter very much vulnerable to the passage of an electron associated with the nuclear-active particle and thus confusing the issue

4 A large value of herght decreases the aperture drastically (Large apertures are imperatıve because of the very low beam intensities )

Any effort to alleviate the difficulties 1 and 2 above by increasing the height of the Cerenkov counter would certainly make the matters 3 and 4 worse

\section{Methods Based on RELATIVISTIC RISE OF ENERGY LOSS}

It is well known that the energy loss of a particle ncreases $^{\dagger}$ with its energy at energies $\geq 2 m c^{2}$ Since the energy loss is a function of only the Lorentz factor, $\gamma$, of the incident particle (charges of pion and proton being equal), a measure of energy, $\gamma m^{2}{ }^{2}$, in a calorimeter or momentum, $\left(\gamma^{2}-1\right)^{\frac{1}{2}} m c$, in a magnetic field and the energy loss. $f(\gamma)$, determine the mass $m$, of the particle Solids are not suitable for this approach since the saturation in the energy loss due to the density effect sets in at too low a value of $\gamma$ to be of any use to us Three different techniques have been mooted in this connection (a) $\mathrm{Yuan}^{3}$ ) and Purcell ${ }^{4}$ ) discuss the possibility of using xenon scintillation counters, (b) Dobrotın et $\mathrm{al}^{5}$ ) and Cowan et $\mathrm{al}^{6}$ ) consider the possibility of utılizing the drop count technique in cloud chambers and (c) we ourselves are trying the idea of using an array of gas proportional counters

Though the average and most probable energy losses in a medium of a given thickness increase with the Lorentz factor of the incident particle, this increase is smaller than the Landau fluctuations which inevitably exist even at a constant Lorentz factor Therefore making just one observation of energy loss does not

* We do not go here into the detalls such as what gases, under what pressures, do have these desired values of refractive indices

The relativistic rise is partly due to increase in the value of maximum transferable energy and partly due to the relativistic expansion of the zone of electrical influence of the incident particle It is with the latter effect we are concerned here 
help to distinguish the particles (In this respect drop count technique is different from the others to which we refer later on.) The problem cannot be solved by simply increasing the pathlength of the particle in the detector since it is in the very nature of Landau fluctuations that the greater the pathlength the greater is the probability of occurrence of rarer and higher energy transfers; consequently the fractional width of Landau distribution defined as the ratio of full width at half maximum to the most probable energy loss becomes almost independent of the pathlength One can sample the energy losses in several individual counters and by means of a suitable statistical analysis of the energy loss measurements in individual counters, one can obtain a much narrower distribution The smaller the width of the resulting distribution, compared to the expected rise in the energy loss, the better are the changes of distınguishing the particles

\section{2.1. Gas scintillation counters}

The light output from a gas scintillation counter is proportional to the energy deposited by the incident particle, thus a measure of the intensity of light is a measure of the energy loss However, the light output, even when the best of the gases - namely xenon-is used, is too low Under ideal circumstances of operation and with light collection efficiency (at the photocathode of the photomultiplier) of near unity, experiments ${ }^{7}$ ) indicate that an energy loss of $2 \mathrm{keV}$ by the incident particle results in 1 photoelectron at the photocathode To keep the error due to photoelectron statıstics much smaller than Landau fluctuations one has to collect $\approx 1000$ photoelectrons which implies trajectories $\approx 200 \mathrm{~cm}$ in xenon at 1 atm pressure If the energy losses were to be sampled in 6 or 12 counters to perform a statistical analysis to get around Landau fluctuations, one has to use 12 to 24 meter of xenon gas Such pathlengths lead to the same difficulties as pointed out earlier in section 21 (3 and 4) When Yuan ${ }^{3}$ ) and Purcell $^{4}$ ) discussed this 1dea, it was to distinguish the particles in the accelerator beams which are well collimated, and hence the difficulties mentioned here do not apply On the other hand, because of the high beam intensities, the counting system must have a short rise and recovery tıme and this technique has an advantage

The essential difference between an ideal gas scintillation counter and a gas proportional counter, which will be considered later on, is that about $2000 \mathrm{eV}$ of energy is required to give rise to one photoelectron from a scintillator while about $26 \mathrm{eV}$ is sufficient to produce one ion pair in a gas proportional counter
This feature obviously renders the proportional counter statistically more efficient for a given energy loss

\section{2. Drop count technique in cloud chambers}

In this technique, while counting the droplets condensed around lons along the path of a charged particle in a cloud chamber one rejects clusters of greater than a particular size By doing this, one is measuring a quantity that is proportional to that part of energy loss which consists of all encounters with energy transfers, $E^{\prime}$, less than a particular value, usually chosen around $1000 \mathrm{eV}$ Since such encounters in a pathlength of $40 \mathrm{~cm}$ of argon or xenon are many, one may apply a Gaussian distribution rather than the Landau type to the fluctuations in ionization

Referring to our specific problem of distınguishing a $100 \mathrm{GeV}$ proton from a $100 \mathrm{GeV}$ pion, Sternheimer's equations $^{8}$ ) predict that the proton loses an energy of $848 \mathrm{keV}$ and the pion $946 \mathrm{keV}$ (both with the restrictıon $E^{\prime}<1 \mathrm{keV}$ ) over a pathlength of $40 \mathrm{~cm}$ in a chamber filled with argon at one atmospheric pressure Takıng the value $264 \mathrm{eV}$ for the average energy loss per ion pair in argon as determined by Jesse and Sadauk1s $^{9}$ ), the two tracks will have 3210 and 3590 droplets respectively These numbers represent probable specific ionization It is more appropriate to take for the statistical errors applicable to these numbers the one determined by the primary specific ionization which is approximately half the probable specific ionization The statistical error, then, is of the order of $\pm 255_{0}^{\circ}, 1 \mathrm{e}, 80$ droplets The expected difference being about 380 droplets, probability that a pion will be confused for a proton and vice versa is extremely small

A definite advantage this method has over the others is that one can tell whether the particle is a pion or is a proton Recall that with the threshold Cerenkov technique, one can be sure about only one of the two species The disadvantages are that it is a slow and tedious technique (imagine counting the droplets over 50000 to 100000 tracks, each consisting of more than 3000 droplets'), and constant attention has to be paid to ensure that the efficiency of condensation on ions is $100 \%$ throughout the experiment

\section{Proportional counters}

Ionization caused by the incident charged particle in a proportional counter is proportional to the energy it lost (except in the rare cases where a knock-on electron might be so energetic as to leave the counter without dissipating all its energy), and therefore a measurement of ionization is a good measure of energy 


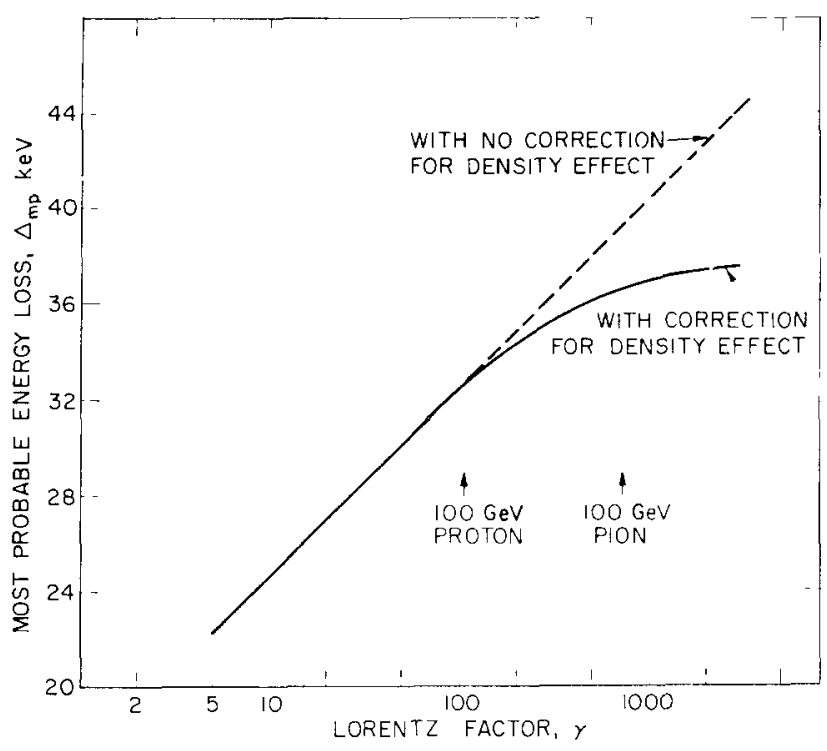

Fig 1 Most probable energy loss in $15 \mathrm{~cm}$ of argon gas at one atmospheric pressure, suffered by an incident singly charged particle with Lorentz factor $\gamma$ is shown as a function of $\gamma$

loss. We plan to use the proportional counters rather than 1on chambers in order to take advantage of gas multiplication to present at the grid of the first stage of the preamplifier a significantly large signal compared to the equivalent input noise Dimensions of the individual proportional counters in the vertical plane should be 1 as small as possible in the interest of a compact geometry for a good aperture and fast collection of electrons, but 2 large enough so that even for a constant given energy loss the fluctuations in the number of ion parrs are small compared to the fluctuations in the energy losses. A herght of 10 to $15 \mathrm{~cm}$ should be a suitable compromise if the counter is filled with argon at about one atmospheric pressure We have built and tested several proportional counters with dimensions of about 10 to $15 \mathrm{~cm}$ in the vertical plane, the performance of which will be described in section 3 In this section we shall base all of our considerations on a $15 \mathrm{~cm}$ proportional counter filled with argon at one atmospheric pressure

In fig 1 , we have shown the calculated most probable energy loss as a function of the Lorentz factor, $\gamma$, based on the formula given by Rossi ${ }^{10}$ ) Correction due to the density effect, which becomes applicable at $\gamma \gtrsim 100$ in the present case, is also shown in the figure This correction is calculated on the basis of formulae given by Sternheimer ${ }^{8}$ ) It is seen that the most probable energy losses for a proton and a pion both of $100 \mathrm{GeV}$ energy are 331 and $367 \mathrm{keV}$ respectively

Theoretical distributions of energy losses suffered by an incident particle of a given initial energy have been given by Landau ${ }^{11}$ ), by Symon ${ }^{12}$ ) and more recently by Vavilov ${ }^{13}$ ) While these various distributions differ

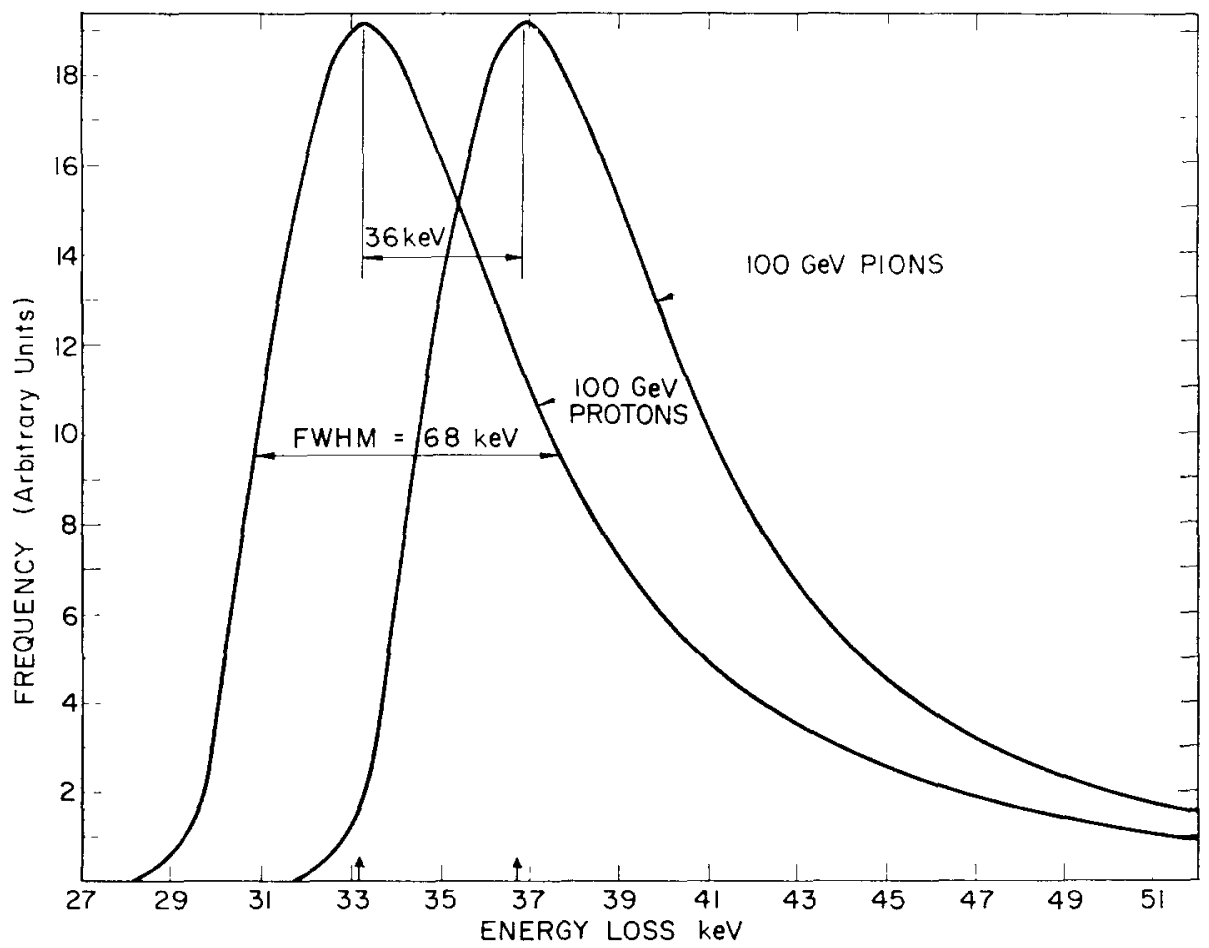

Fig 2 Landau distributions of energy loss in $15 \mathrm{~cm}$ argon (pressure $1 \mathrm{~atm}$ ) suffered by $100 \mathrm{GeV}$ protons and $100 \mathrm{GeV}$ pıons 
in the cases of moderately thick absorbers and for near relatıvistic particles $(\gamma \gtrsim 1)$, there is practically no difference between them for the case of ultra-relativistic particles $(\gamma \gg 1)$ traversing thin detectors which applies to our experiments. In fig 2 , we have shown the fluctuations in the energy losses suffered in the counter by pions and protons both of $100 \mathrm{GeV}$ energy These curves are drawn from the tabulated values given by Seltzer and Berger ${ }^{14}$ ) on the basis of the theory of Vavilov ${ }^{13}$ ) It is seen that there is a considerable amount of overlap, and a single measurement seldom distinguishes a proton from a pion. As stated earlier the situation cannot be improved just by increasing the pathlength using but a single counter The way seems to be to employ an array of $N_{0}$ counters where $N_{0}$ is large enough a number and devise an optımal statıstical procedure to distınguish the particles. The following methods may be considered.

a Igo and Eisberg ${ }^{15}$ ) built an array of 3 proportional counters, exposed them to a beam of $31 \mathrm{MeV}$ protons and showed that the distribution of the smallest of the three pulse heights from the three counters has, as expected, a much narrower width than that of the pulses from any individual counter.

b Since the Landau distribution is skew with a long tall, one can impose a cut-off on the side of higher energy losses (1.e, ignore the information from those counters which showed a pulse height greater than a chosen value), compute the average of these restricted energy losses, and see if the resulting distribution of the restricted average energy losses is narrow enough to resolve protons and pions

c The third method is the one suggested by Purcell ${ }^{4}$ ) In this, if one demands that in at least $N$ out of the $N_{0}$ counters the pulse height must be smaller than a chosen value, one favors the selection of protons, strongly discrimınatıng against pions

d. The fourth is a likelihood ratio method, here one computes the ratio

$$
L=\prod_{i=1}^{N_{0}} y_{t, \text { proton }} / \prod_{i=1}^{N_{0}} y_{\imath, \text { pion }},
$$

where $y_{i, \text { proton }}$ is the value of the ordinate of the Landau curve for protons at the pulse height $\Delta_{\imath}$ from

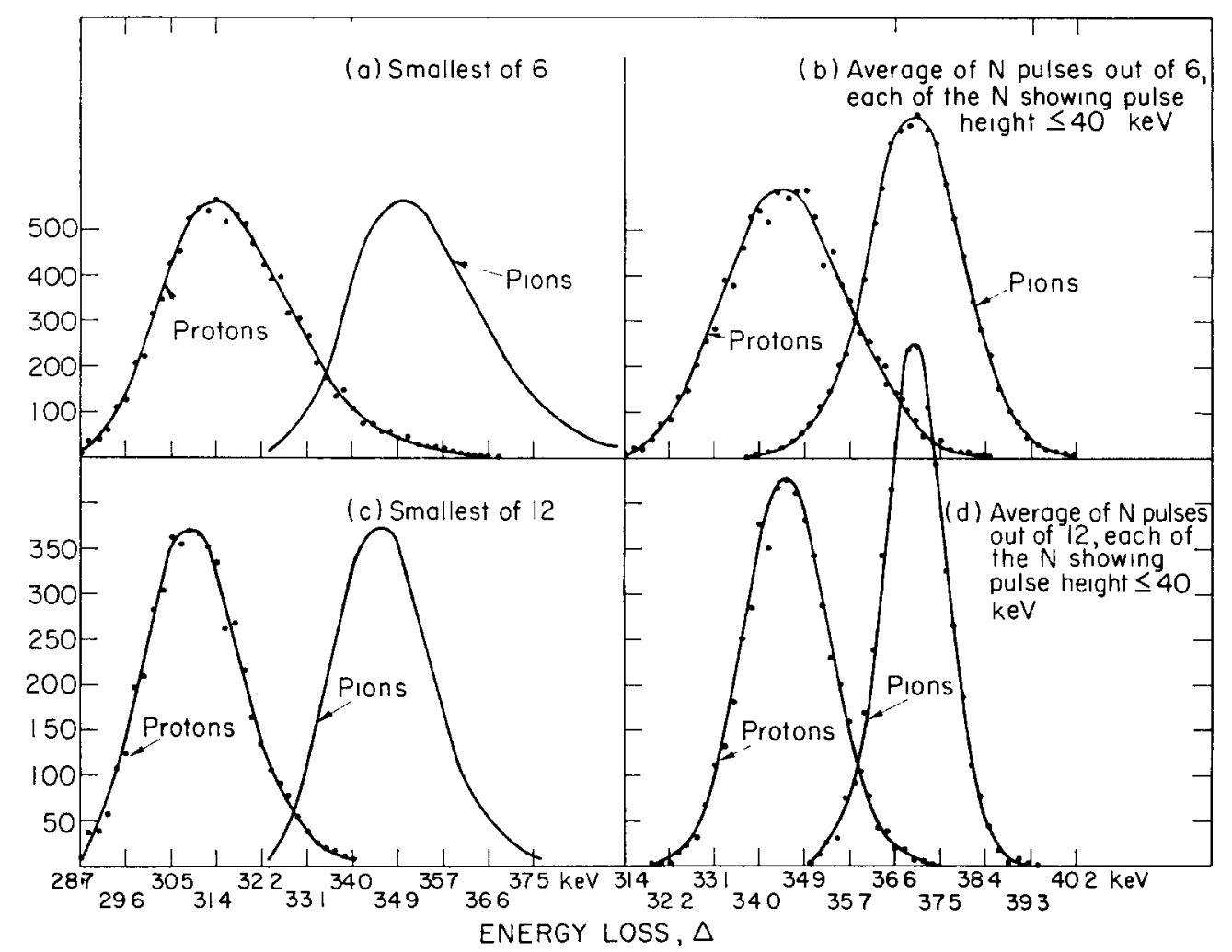

Fig 3 This figure is based on the assumption that each of $N_{0}$ detectors show the Landau distribution shown in fig 2 Shown in the figure are the distributions obtained from Monte Carlo calculations of the smallest and average pulse heights in the arrays of $N_{0}=6$ and 12 detectors In each case, distribution to the left corresponds to protons and to the right to pions, both of $100 \mathrm{GeV}$ energy While computing the averages, an arbitrary cutoff is made at $40 \mathrm{keV}$ and information from counters showing pulse height greater than this value is ignored Points are the results from the Monte Carlo calculations and curves are drawn through the points. 


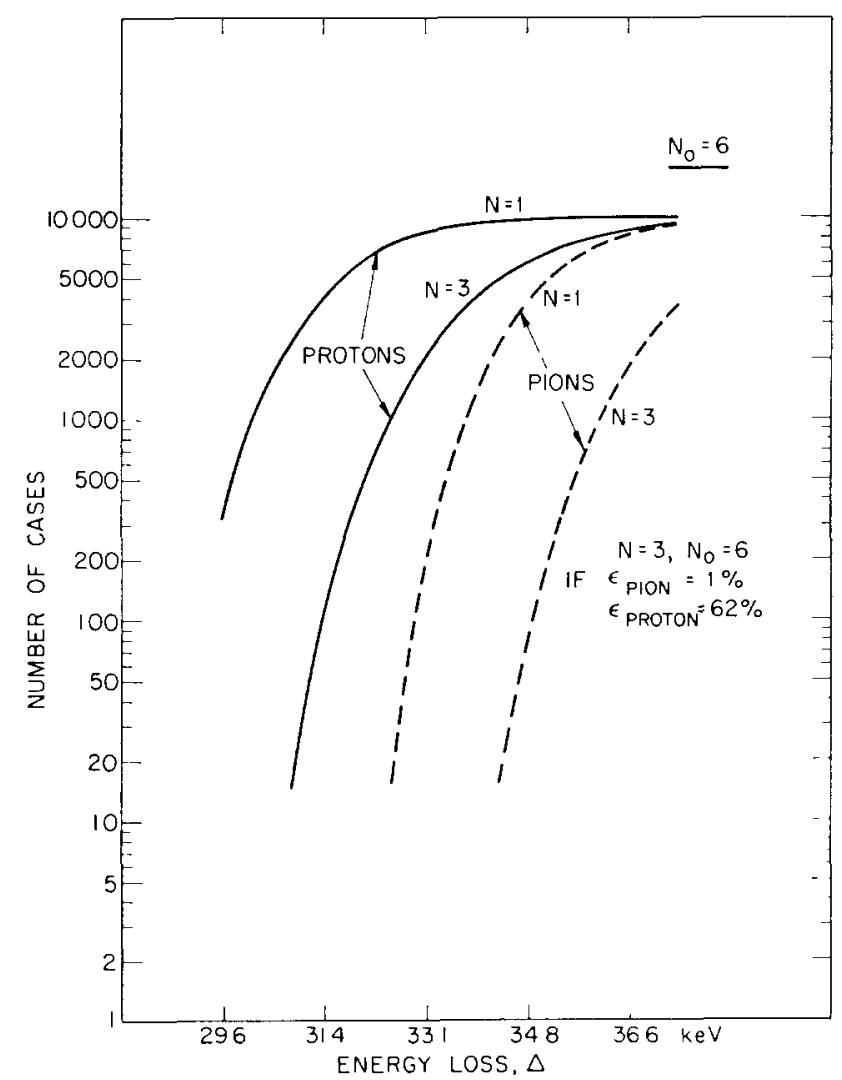

Fig 4 Results from Monte Carlo calculations on the basis of Landau distribution of the energy losses (fig 2) for 10000 protons and 10000 pions incident on an array of 6 detectors Numbers of cases in which at least $N$ out of six counters showed energy losses $<d$ is shown as a function of 1

the $t^{\text {th }}$ counter and $y_{1, \text { pion }}$ the corresponding quantity for pions If this ratio, $L$, is $\gg 1$, the incident particle is a proton and if $L \ll 1$, it is a pion If $L$ happens to be $\approx 1$, there will be some ambiguity and the degree of ambiguity depends on how well the $L$ values for the assumed incidence of a pure proton beam and for a pure pion beam separate

To test which of these approaches is most efficient, the following is done An empirical formula, modified from the one given by Moyal ${ }^{16}$ ), of the type

$$
\begin{array}{r}
F(\Lambda) \mathrm{d} \Lambda=0762(2 \pi)^{-\frac{1}{2}} \exp \left\{-05\left(\Lambda+\mathrm{e}^{-1}\right)\right\} \mathrm{d} \Lambda, \\
\text { for } \Lambda \leqq 0, \\
=0762(2 \pi)^{-\frac{1}{2}} \exp \left\{-05\left(\Lambda^{085}+\mathrm{e}^{-\Lambda}\right)\right\} \mathrm{d} \Lambda, \\
\text { for } 0<A<10,
\end{array}
$$

is chosen to represent the Landau distribution shown in fig 2 Here $A$ is a dimensionless parameter, and, in its terms, any arbitrary energy loss, $\Delta$, in the counter is expressed by

$$
\Delta=\Delta_{\text {most probable }}+\Lambda\left\{0300 m c^{2}(Z / A) x\right\},
$$

where

$m=$ rest mass of the electron,

$Z=$ atomic number of the medium,

$A=$ atomic weight of the medium,

$x=$ thickness of the gaseous medium measured in $\mathrm{g} / \mathrm{cm}^{2}$

Assuming that the incident particle is a proton and using eq (7) as the reference curve, a set of $N_{0}$ random pulse heights (called here as one proton event) are generated on the computer according to the method suggested by Von Neumann ${ }^{17}$ ). We have chosen an array of 6 and 12 counters $\left(1 \mathrm{e}, N_{0}=6,12\right)$ A total of 10000 proton and 10000 pion events with $N_{0}=6$ and 5000 pion events with $N_{0}=12$ is generated on the computer and analyzed with the methods (a), (b), (c) and (d) mentioned above

The results for (a) and (b) are shown in fig 3 As can be seen from the figure, the resolution between the protons and pions has improved considerably The choice of the smallest of 12 pulse heights seems to be

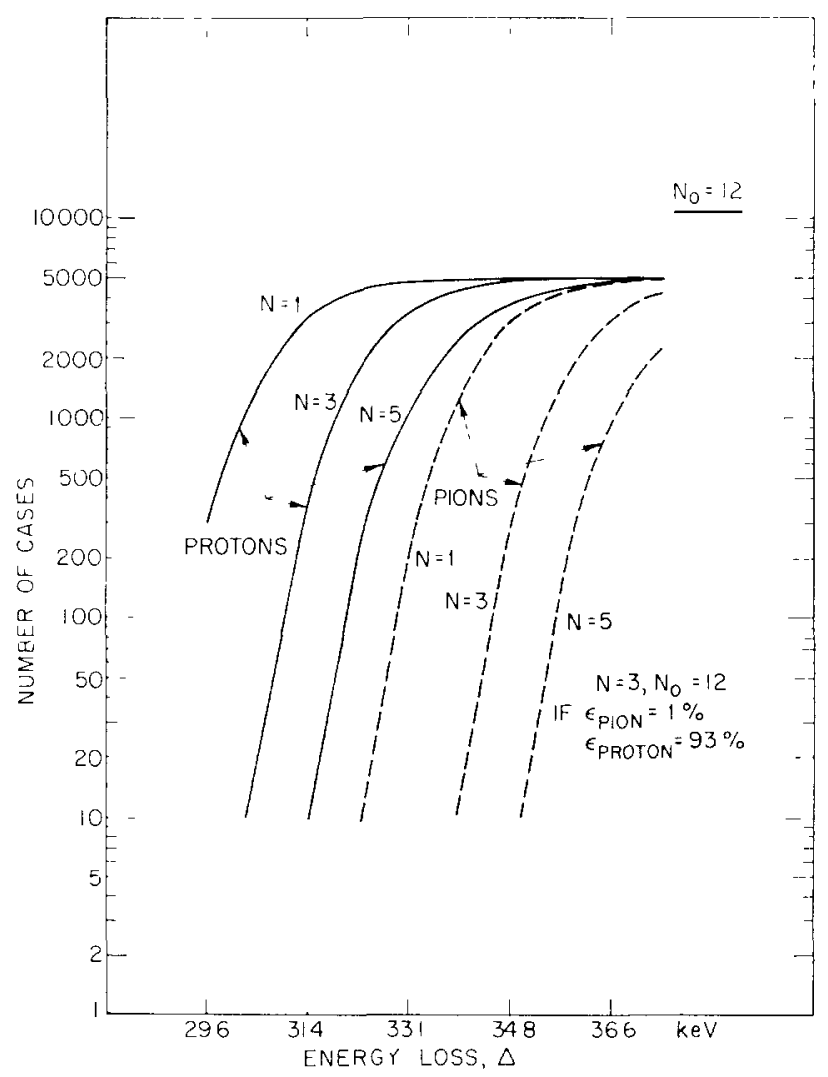

Fig 5 Same as in fig 4 but for 5000 protons and 5000 pions incident on an array of 12 detectors 
the most effective means of separating the particles For example, if one demanded that the smallest of the 12 be smaller than $\Delta_{0}=32.4 \mathrm{keV}$, one would count protons with $93 \%$ efficiency and pions with $08 \%$ efficiency. Since the $\pi^{+} / p \approx 02$ to 04 in the cosmic radiation at mountain altitudes and at energies of a few hundred $\mathrm{GeV}$, these figures would mean that we have a selected proton beam of $99.7 \%$ purity.* On the other hand, by demanding that the smallest pulse height from the 12 counters be greater than $\Delta_{0}=34$ $\mathrm{keV}$, one would count protons with an efficiency of $04 \%$ and pions with $73 \%$ efficiency; the selected pion beam will be $98 \%$ pure. To increase the purity of the $\pi^{+}$beam, one has to increase the value of $\Delta_{0}$, in which case one has to forego part of the pion intensity

Results for the approach (c) are shown in fig. 4 (for $N_{0}=6$ ) and in fig 5 (for $N_{0}=12$ ) Let us spell out two examples By insisting that in each of at least three

* This is so if one assumes kaons are totally absent which is unlikely In the presence of kaons, $97 \%$ seems to be the limit of purity of a beam

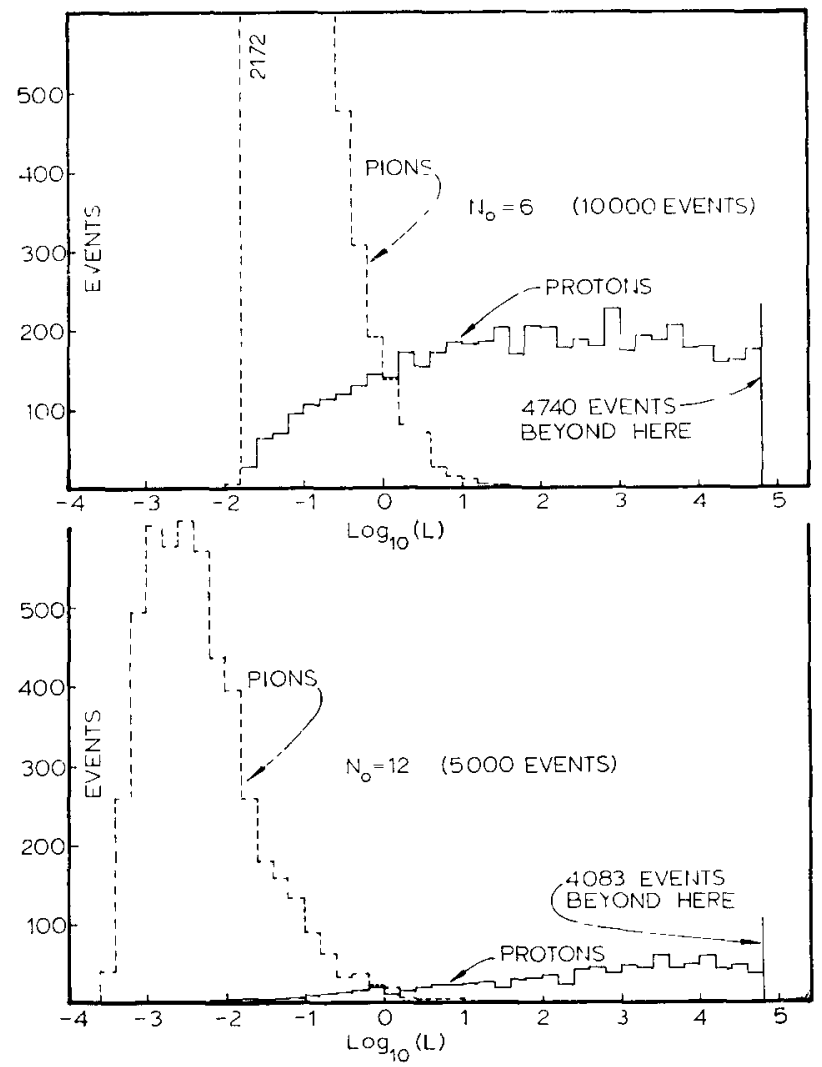

Fig 6 Frequency distribution of likelihood ratios for 10000 proton and 10000 pion events in an array of 6 counters and for 5000 proton and 5000 pion events in an array of 12 counters These ratios are computed for artificially generated events obeying theoretical Landau distributions
TABLE 1

Efficiency for counting protons when conditions are so set as to count pions with $1 \%$ efficiency

\begin{tabular}{lcc}
\hline $\begin{array}{l}\text { Number of counters in the array, } N_{0} \\
\text { Smallest pulse from the } N_{0} \text { counters should be }\end{array}$ & 6 & 12 \\
smaller than $\Delta_{1}{ }^{*}$ & $81 \%$ & $95 \%$ \\
At least 3 out of $N_{0}$ counters should show pulse & & \\
heights less than $\Delta_{2}{ }^{*}$ & $62 \%$ & $93 \%$ \\
Likelihood ratio, $L>L_{1}{ }^{*}$ & $89 \%$ & $98 \%$
\end{tabular}

* $\overrightarrow{\Delta_{1}, \Delta_{2}}$, and $L_{1}$ are chosen in each case to count pions with $1 \%$ efficiency

out of six detectors the pulse heights be less than 34.8 $\mathrm{keV}$, one can count protons and pions with efficiencies of $60 \%$ and $0.8 \%$ respectively. If one insists that in at least three out of twelve detector's the pulse heights be less than $342 \mathrm{keV}$, one counts protons and pions with efficiencies of $93 \%$ and $08 \%$ respectively $\mathrm{Ob}-$ viously, having 12 detectors is better, and efficiencies for selection of one species and rejection of the other are about the same as in (a)

Results for the method (d) are shown in fig. 6 . Separation of $L$ values for the incident proton and pion beams is not complete, though it is much better than in the methods (a), (b) and (c) As an example, if one considered only those events which show $L>3$, one counts with an array of 12 counters protons with $973 \%$ efficiency and pions, with $036 \%$. Referring to the $\pi^{+} / \mathrm{p}$ ratio of the order of $0.3 \mathrm{in}$ cosmic radiation, these figures would mean the following If an unknown positive particle of energy of $100 \mathrm{GeV}$ (as measured in a calorımeter or a magnet spectrograph) is incident on the array of 12 proportional counters and if the value of $L$ computed from the 12 pulse heights happened to be $>3$, one can say that the incident particle has a $999 \%$ probability of being a proton and a $01 \%$ probability of being a pion. Simılarly, one can have a selected pion beam by demanding $L<02$ or so.

Relative merits of the various methods can be gauged from the example in table 1 above [method (b), being obviously inferior to the others, is not considered in the table]

Presented in the table are the efficiencies for counting the protons when conditions are so chosen as to count pions with $1 \%$ efficiency. Likelihood ratio method, being capable of counting protons with highest efficiency for a given efficiency for pions, is the most efficient.

All of the discussion so far has been based on the assumption that, at any given energy of the incident particle, the distribution of the energy losses in the counters obeys the theoretical Landau distribution. Experimentally, it is found that this is not so, and the 
distributions in reality are much wider at the energies of our interest than the theoretical Experiments leading to this conclusion and its effect on the separability of protons and pions are discussed in section 3

\section{Experiments with proportional counters}

To pursue the problem experimentally, we have built and tested a number of proportional counters of the following dimensions
a $15 \times 15 \times 200 \mathrm{~cm}^{3}$ (square cross-section),
b $15 \times 15 \times 40 \mathrm{~cm}^{3}$ (square),
c $75 \mathrm{~cm}$ dia $\times 65 \mathrm{~cm}$ long (cylindrical),
d $15 \times 40 \times 40 \mathrm{~cm}^{3}$ (rectangular);
e $15 \times 100 \times 200 \mathrm{~cm}^{3}$ (rectangular)

Of the above, types (a), (b) and (c) had a single anode wire stretched at the center and (d) and (e), several wires in the mid plane, one every $5 \mathrm{~cm}$ Most of the tests were made with anodes of $0005^{\prime \prime}$ dia steel wire Pure argon gas and mixtures of argon with $\mathrm{CO}_{2}, \mathrm{~N}_{2}$

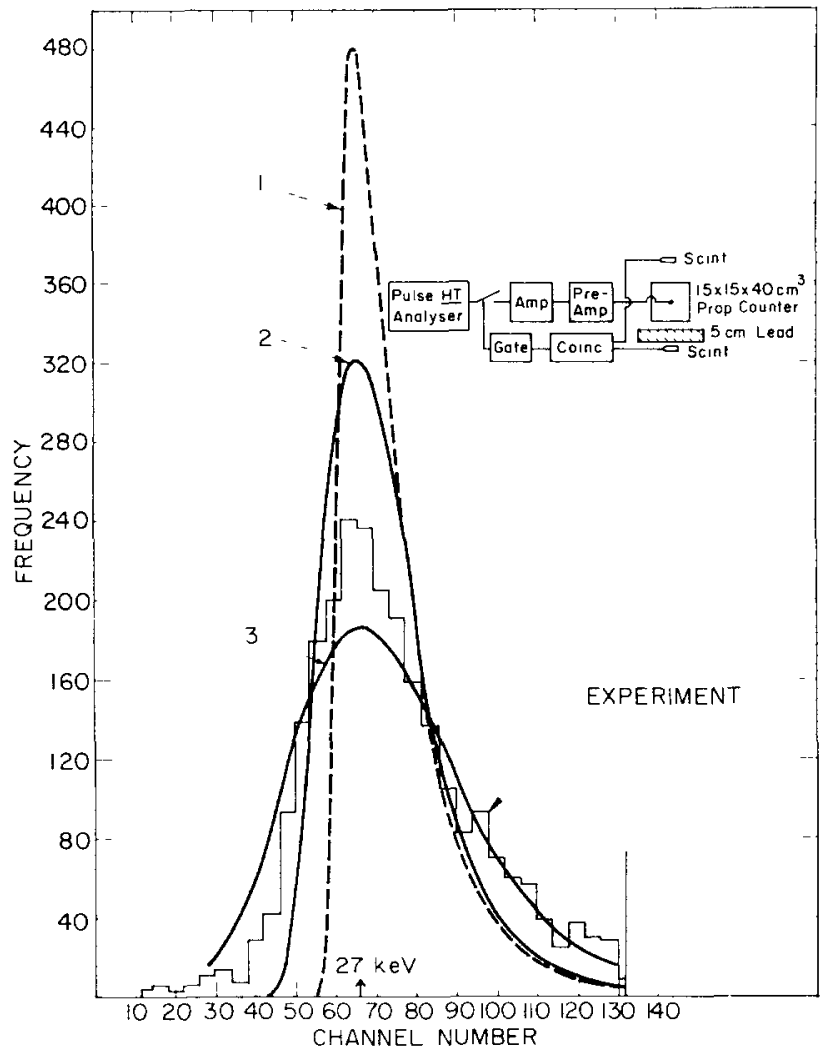

Fig 7 Experimental results on the distribution of energy losses of cosmic rays at sea level in a pathlength of $15 \mathrm{~cm}$ in the proportional counter are shown Curve 1 is the calculated theoretical Landau distribution assuming muons are monoenergetic, curve 2, the same but considering the spread in energy of muons and curve 3, Blunck and Leisegang distribution for the spectrum of muons

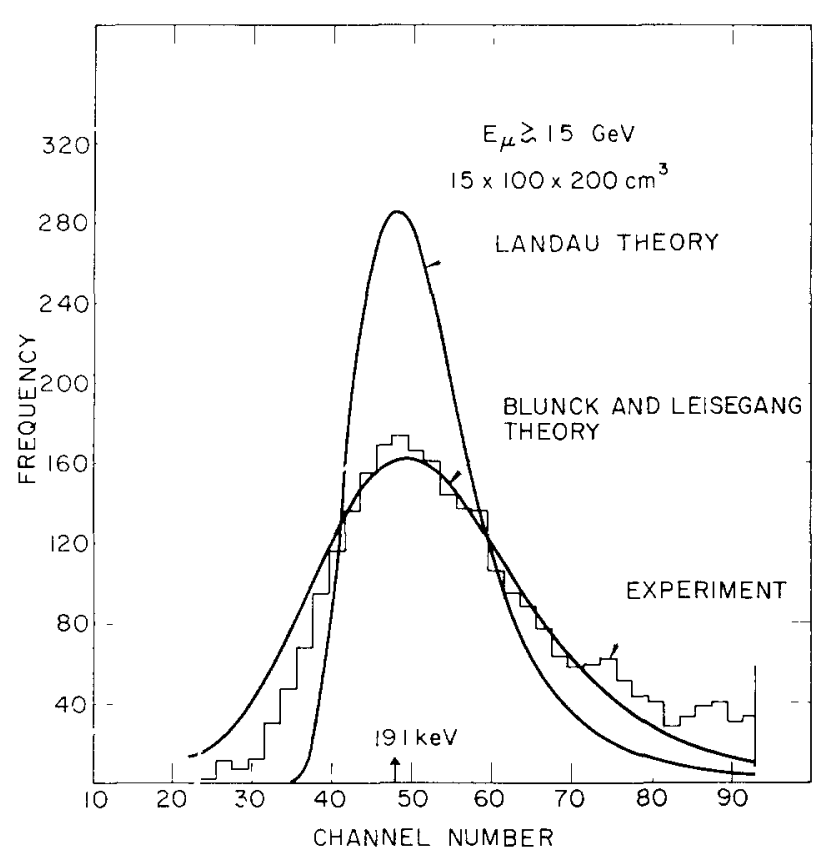

Fig 8 Frequency distribution of energy losses suffered by cosmic ray muons at $\mathrm{Mt}$ Evans in a pathlength of $15 \mathrm{~cm}$ in the proportional counter Smooth curves are those calculated on the basis of theories of Landau and of Blunck and Leisegang for all the muons above a low energy cut-off at $15 \mathrm{GeV}$ determined by the absorber (calormeter) in the beam definıng telescope

and $\mathrm{CH}_{4}$ were tried as the filling, and it was considered that the mixture of $93 \%$ argon and $7 \%$ methane was the best because, with this mixture, the rise times of the pulses were shortest ( 15 to $2 \mu \mathrm{sec}$ ) and gas mult1plication factors, $M$, highest at a given voltage in a given counter All the counters were tested with cosmic ray muons to see if the distributions of pulse heights from the proportional counters, gated by coincidences from narrow angle cosmic ray telescopes, agree with the theoretical distributions of energy losses Since cosmic ray muons have varying energies, the Landau distribution is folded into the energy spectrum of muons before a comparison is made with exper1mental results

Before presentıng the experimental results, we might mention that we have considered in detail the problem of energetic secondary electrons leaving the proportional counter without dissipating all their energy This has two effects on the observations. Firstly, the ionization in the counter underestımates the actual energy losses thereby makıng the experımentally determıned distribution of energy losses narrower than the true Landau curve Secondly it introduces a correlation in pulse heights recorded by two or more consecutive layers of proportional counter arrays in that if 
one layer records a pulse height larger than usual due to production of knock-on electrons, the layer below will also record a pulse larger than the usual It is calculated that this sort of thing happens in less than
$15 \%$ of the traversals, for counter dimensions used in this study Closely related to this phenomenon is the possibility of knock-on electrons produced in the walls of the counter entering the counter thus making the

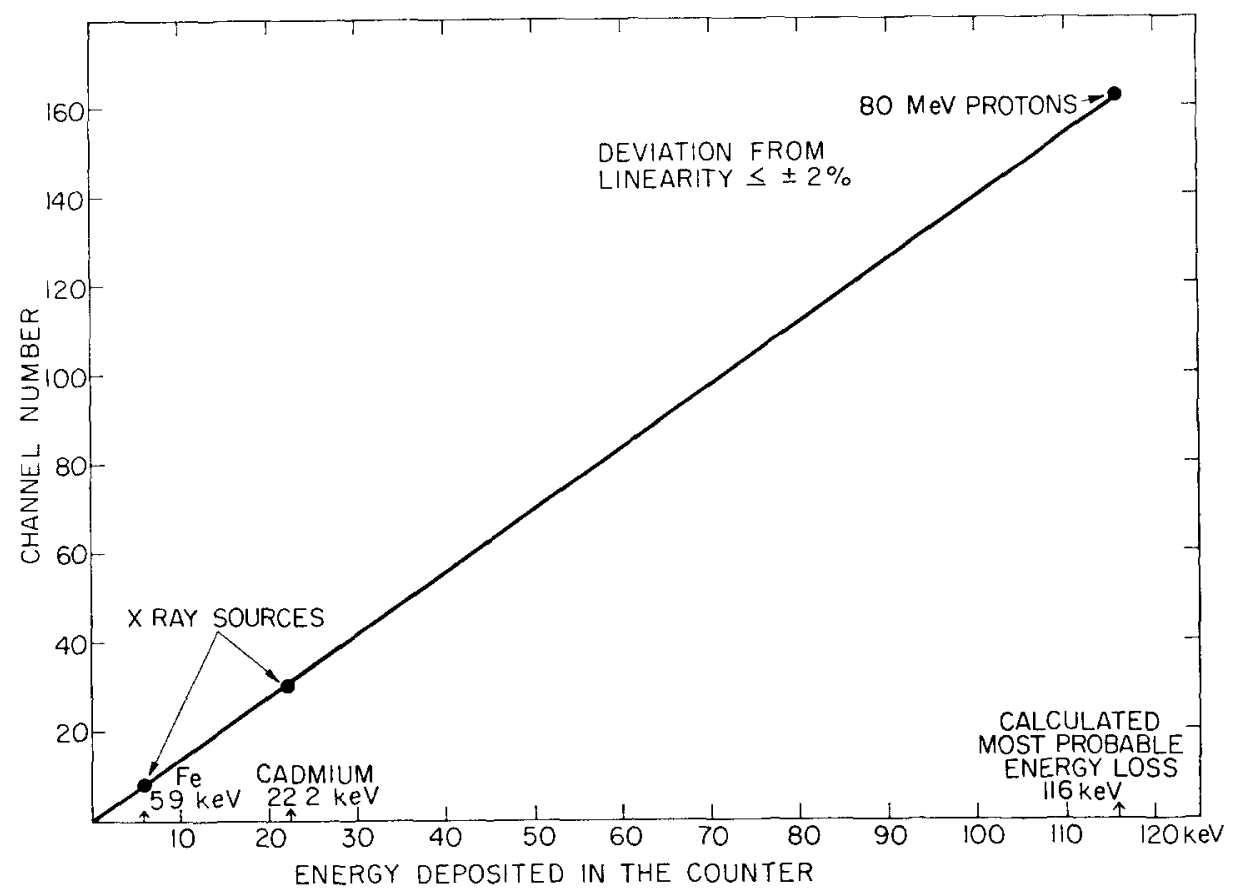

Fig 9 Linearity of the $15 \times 15 \times 40 \mathrm{~cm}^{3}$ proportional counter for energy losses in the range 5 to $120 \mathrm{keV}$ The counter is operated at $2370 \mathrm{~V}$ and the approximate value of the gas multiplication factor is 75

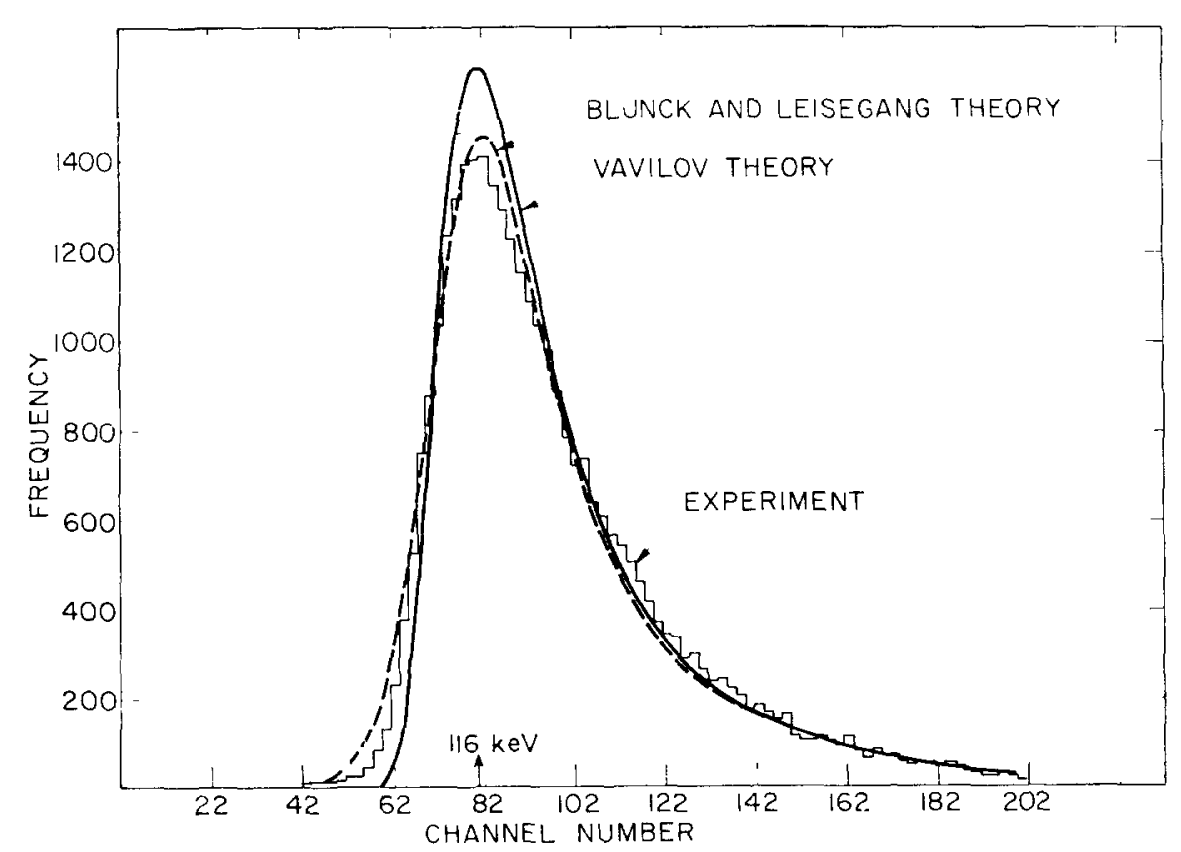

Fig 10 Frequency distribution of energy losses suffered by $80 \mathrm{MeV}$ protons in a pathlength of $15 \mathrm{~cm}$ in the proportional counter Smooth curve is the distribution based on Vavilov's theory and the dashed curve on the Blunck and Leisegang theory 


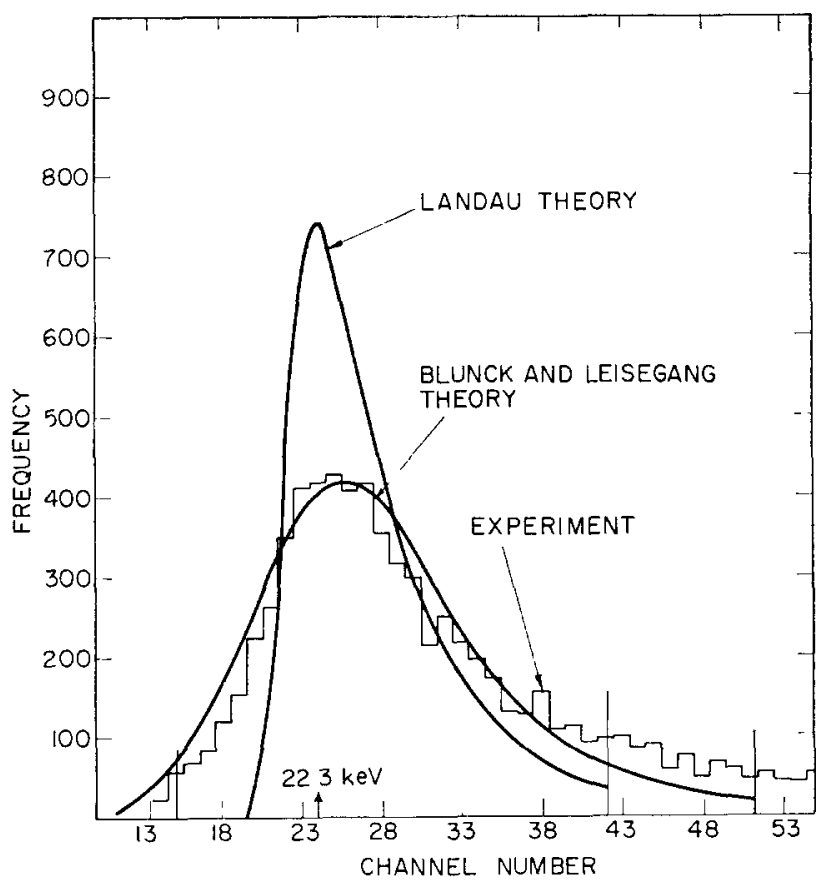

Fig 11 Same as in fig 10 , but for an incident $15 \mathrm{GeV} \pi^{-}$beam.

apparent energy loss in the counter bigger than what it really $1 \mathrm{~s}$. The probability of this happening is again low for wall thicknesses $<\frac{1}{8}$ " of aluminum We had exper1mentally obtained two distributions with $40 \mathrm{GeV}$ pion beams, one with a wall thickness of $\frac{1}{16}$ " aluminum and the other with a wall thickness of $0001^{\prime \prime}$ of aluminum keeping all other conditions the same and found no difference between the two distributions. This, in agreement with our calculation, clearly shows that leakage of electrons into or out of the counter is a phenomenon infrequent enough that we can ignore it

Typical results from cosmic ray muon tests are shown in figs 7 and 8 . Invariably, the experimental distributions are all wider than those expected theoretically. In an effort to understand the reasons for this discrepancy, counters (b) and (c) were subjected to closer examination These two counters were tested with X-rays from ${ }^{55} \mathrm{Fe}(59 \mathrm{keV})$ and ${ }^{109} \mathrm{Cd}(222 \mathrm{keV})$, with $80 \mathrm{MeV}$ protons $(\gamma=1.085)$ from the cyclotron at the Lawrence Radiation Laboratory, Berkeley, and with negative pions of energies $15 \mathrm{GeV}(\gamma=107)$ and $40 \mathrm{GeV}(\gamma=28.6)$ at the Bevatron, also at the Lawrence Radiation Laboratory. Experımentally measured resolutions of the counters at X-ray energies are in reasonably good agreement with the values expected on the basis of Gaussian distribution of numbers of Ion pairs Proportionality is good withın $\pm 2 \%$ over a wide range, as shown in fig 9. A typical pulse height distribution taken with $80 \mathrm{MeV}$ proton beam (pulses are gated by beam definıng coincidence telescopes) is shown in fig. 10 along with theoretically expected distribution calculated from the tabulated values given by Seltzer and Berger ${ }^{14}$ ) based on Vavilov's theory ${ }^{13}$ ) The agreement seems to be satisfactory* Pulse distr1butions taken with $15 \mathrm{GeV}$ and $4 \mathrm{GeV}$ pion beams are shown in figs. 11 and 12 respectively As can be seen from the figures, there is no agreement between theory and experiment The situation at first appeared paradoxical in that a proportional counter showing good linearity and expected resolution with X-ray sources yields pulse height distributions in agreement with theoretical predictions in the case of $80 \mathrm{MeV}$ proton beams but not in the cases of $15 \mathrm{GeV}$ and $4 \mathrm{GeV}$ pion beams On a closer look, however, it became clear that the theoretical distributions as given by Landau ${ }^{11}$ ), Symon ${ }^{12}$ ) and Vavilov ${ }^{13}$ ) in which the effects of atomic binding of electrons are not taken into account do not apply to the cases of pion and muon beams traversing thin proportional counters Taking these atomic binding effects into account, Blunck and Leisegang ${ }^{18}$ ) and Blunck and Westpha $1^{19}$ ) modified the Landau theory and showed that, depending on the experimental situation, the distributions could be much broader than those given by Landau. Definıng a parameter $b^{2}$ as

$$
b^{2}=\vec{\Delta}(\mathrm{eV}) Z^{\frac{4}{3}} \times 20(\mathrm{eV}) / \xi^{2}(\mathrm{eV})^{2},
$$

* It may be mentioned that the measured noise of the preamplıfier-amplıfier system, being only $06 \%$ of the most probable pulse height did not affect the measured widths with protons

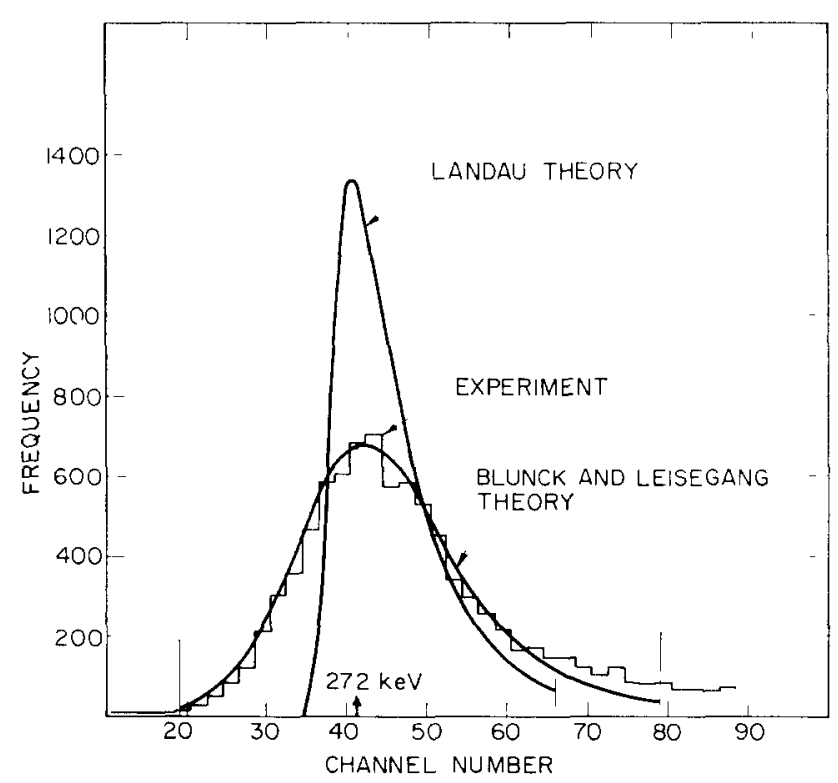

Fig 12 Same as in fig 10 , but for an incident $40 \mathrm{GeV} x^{-}$beam 
where

$\bar{\Delta}=$ average energy loss in traversing the counter of $x \mathrm{~g} / \mathrm{cm}^{2}$,

$Z=$ atomic number of the medium,

$\xi=0300(Z / A) x\left(m_{\mathrm{e}} c^{2} / \beta^{2}\right)$,

$m_{\mathrm{e}}=$ mass of electron,

$\beta c=$ velocity of the incident particle,

these authors come to the conclusion that when $b^{2} \ll 3$, no correction need be applied to the original Landau distribution and when $b^{2} \gg 3$, the true distribution is much wider than the one given by Landau* Indeed, in our experiment, $b^{2}=1.184$ for the case of $80 \mathrm{MeV}$ proton beams where there is not much difference between Vavilov ${ }^{13}$ ) and Blunck and Lersegang ${ }^{18}$ ) theories and our experiment agrees with both the theories (fig 10), and $b^{2}=13$ to 16 for negative pions and cosmic ray muons for which the experimental distributions, though nearly twice as broad as Landau distributions, are in essential agreement with the predictions of Blunck and Leisegang theory (figs $7,8,10$ and 11 )

To test the efficacy of the likelihood ratio test in distinguishing protons and pions at energies $\approx 100$ $\mathrm{GeV}$ with the Blunck and Lelsegang distribution, an empirical formula to fit the Blunck and Leisegang distribution for our experimental conditions is obtained and given below

$$
\begin{aligned}
F(\Lambda) \mathrm{d} \Lambda & =0088 \exp \left(-\Lambda^{2} / 26\right), \quad \text { for } \Lambda \leqq 0, \\
& =0145 \exp \left[-05\left\{\frac{1}{2} \Lambda+\exp \left(-\frac{1}{2} \Lambda\right)\right\}\right] . \\
& \text { for } 0<A<12,
\end{aligned}
$$

where $A$ has the same meaning as defined earler in formula (8). Monte Carlo calculations are repeated with formula (9) as the reference curve instead of the previous formula (7). With these enhanced widths, it becomes, as expected, more difficult to distınguish protons and pions than is implied in figs 3-6 We show as an example in fig 13 , (which is a modification of fig 6 due to enhancement of the widths) the resolution between protons and pions attainable with the likellhood ratio method If one employs an array of 12 layers, it is possible even with the enhanced widths to utilize $60^{\circ}$ of the proton flux at energy $\approx 100 \mathrm{GeV}$ with the confidence that the contamination due to

* This indeed seems to be the reason why in the literature one comes across reports that the energy losses by ultra-relativistic muons or pions recorded in the scintillators $\left(\approx 1 \mathrm{~g} / \mathrm{cm}^{2}\right.$ thick) do obey Landau theoretical distributions whereas those recorded in the gaseous counters $\left(\approx 001 \mathrm{~g} / \mathrm{cm}^{2}\right.$ thick $)$ do not

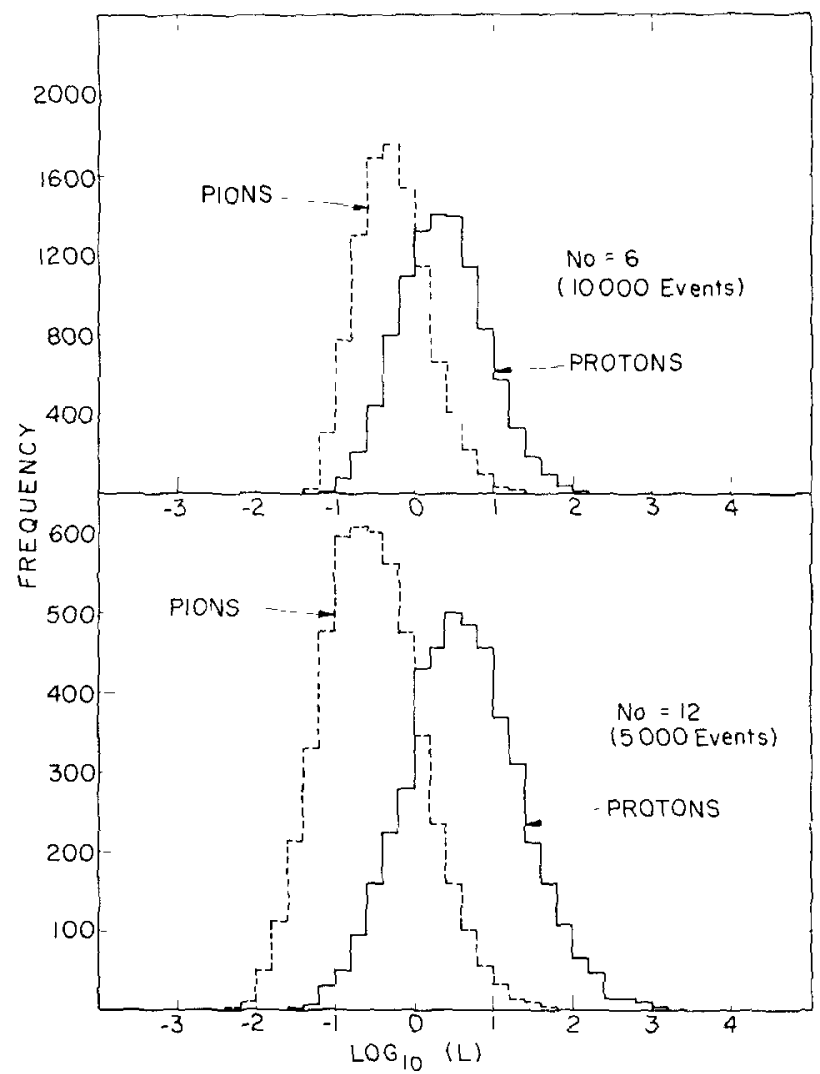

Fig 13 Same as in fig 6 , but the calculations are made on the basis of theoretical Blunck and Leisegang distributions

positive pions is only $\leqslant 3 \%$ in the proton beam so selected.

In table 2 below, we give the separability of protons and pions with an array of 12 counters, using the likelihood ratio method and with the enhanced widths of "Landau" distribution The separability is given in

TABLE 2

Separability of protons and pions in an array of 12 proportional counters by the likelihood ratio method with enhanced widths

To select proton beam

\begin{tabular}{l|r|r|r|r}
$L$ & $\geqq 10$ & $\geqq 30$ & $\geqq 50$ & $\geqq 80$ \\
$\varepsilon_{\mathrm{p}}(\%)$ & 830 & 607 & 503 & 407 \\
$\varepsilon_{\pi}(\%)$ & 192 & 60 & 34 & 18 \\
contamination $(\%)$ & 65 & 29 & 20 & 13 \\
\hline
\end{tabular}

To select positive pion beam

\begin{tabular}{l|r|r|r|r}
$L$ & $<10$ & $<05$ & $<03$ & $<01$ \\
$\varepsilon_{p}(\%)$ & 141 & 69 & 37 & 052 \\
$\varepsilon_{\pi}(\%)$ & 784 & 601 & 481 & 191 \\
contamination $(\%)$ & 374 & 278 & 206 & 83
\end{tabular}


the form of efficiencies for detection of the two kinds of particles, $\varepsilon_{\mathrm{p}}$ and $\varepsilon_{\pi}$

These efficiencies and the $\pi^{+} / \mathrm{p}$ ratio $(\approx 03)$ in the cosmic radiation would imply a certain amount of contamination by the wrong kind of particles whose values are given in the $4^{\text {th }}$ row The separability is asymmetric between pions and protons both because the positive pions are less numerous than protons and because of the asymmetry of the "Landau" curve

We may point out here that the separability of protons and pions is sensitive to the shape of the "Landau" curve to the left of most probable energy loss and to the absolute calibration of the counter output in terms of the energy loss In an actual experiment, both these can be determined with negatively charged nuclear active particles (sense of charge known from the sense of curvature in a magnetic field) in the energy range around $100 \mathrm{GeV}$ To a very good approximation all these particles can be treated as pions An accurate calıbration can also be achieved by using X-ray sources of known energy

\section{Conclusions}

The main results of the considerations discussed in sections 2 and 3 are summarized in table 3 below of all the techniques considered, the drop count method seems to be most accurate. It has the advantages of the best resolution and the least vertical extension affording compact geometries with good apertures and the disadvantage of being a slow and tedious technique This drawback is very serious as it slows down the analysis considerably. While, with this technique, it is possible to identify the particles in individual cases as a demonstration of its power, it appears to the authors that it is impractical to use this technique in experiments planning to collect data on several tens of thousands of particles.

The threshold Cerenkov technique is electronically simple and fast, but its drawback is its enormity in the vertical scale which discourages its use for several reasons discussed in section 2 Multilayer proportional

TABLE 3

A summary of the varıous methods

\begin{tabular}{|c|c|c|c|c|}
\hline \multirow{2}{*}{ Method } & \multirow{2}{*}{$\begin{array}{l}\text { Vertical } \\
\text { extension } \\
(\mathrm{m})\end{array}$} & \multirow{2}{*}{ Speed } & \multicolumn{2}{|c|}{ Identıfication } \\
\hline & & & Protons & Plons \\
\hline $\begin{array}{l}\text { Threshold Čerenkov } \\
\text { Drop count } \\
\text { Prop counter }\end{array}$ & $\begin{array}{l}\approx 5 \\
\approx 05 \\
\approx 15\end{array}$ & $\begin{array}{l}\text { fastest } \\
\text { slowest } \\
\text { medium } \\
\text { fast }\end{array}$ & $\begin{array}{l}\text { poor } \\
\text { good } \\
\text { good }\end{array}$ & $\begin{array}{l}\text { good } \\
\text { good } \\
\text { fair }\end{array}$ \\
\hline
\end{tabular}

counter technique, though electronically more complex, seems to be a suitable compromise between the other two and, in the opinion of the authors, is the only method at the moment suitable to be adapted in a large-scale experıment planning to collect data on several tens of thousands of particles Typically, with this technique, one can utılize nearly $60 \%$ of the 100 $\mathrm{GeV}$ proton flux with the confidence that the contamınation due to pions $<3 \%$ and $48 \%$ of the $100 \mathrm{GeV}$ positive pion flux with the confidence that the contamination due to protons is $\approx 21^{\circ}{ }^{\prime}$. At higher energies $(1 \mathrm{e}, \gamma>1000)$, one has to first establısh experimentally the relativistic rise of energy losses, perhaps with the same apparatus and utılızing the easily identıfiable negative pions, in the light of such experimental information, one can examıne the possibility of exetnding the methods discussed in the paper to distinguish protons and pions to higher energies

The authors wish to thank Professor L W Jones for many useful discussions on this subject and comments on a preliminary version of this note, Mr Orman Haas for fabricating the counters and all other members of the M.U.R A collaboration on the Cosmic Ray exper1ment at Echo Lake, Colorado, US A for their cooperation

One of us (P.V.R M ) would like to express his gratıtude to the Unıversity of Michigan Institute of Science and Technology for its support We would also like to thank Dr Bruce Cork and the staff of the Lawrence Radiation Laboratory, Berkeley, for their assistance and cooperation in the accelerator tests at the Bevatron and the 184-inch cyclotron.

\section{References}

1) S Lal, R Raghavan, B V Sreekantan, A Subramanıan and S D Verma, J Phys Soc Jap 17 Suppl III (1962) 390

2) S J Lindenbaum and L C L Yuan, Methods of Experimental Physics 5A (Academic Press, New York, 1961) ch 15

3) L C L Yuan, High Energy Physics Research (US Govt Printıng Office, Washıngton, D C , 1965) p 449

4) E M Purcell, ibid, p 501

5) N A Dobrotin et al, Proc Int Conf Cosmic Rays (London) 2 (1965) p 817

6) E W Cowan and M K Moe, preprint (1966)

7) We obtained the figure $2 \mathrm{keV} /$ photoelectron from the resolution obtained in a xenon gas scintillator by $\mathrm{A}$ Sayers and C S Wu, Rev Scı Instr 28 (1957) 758, for $\alpha$ particles from 210Po

8) R M Sternheimer, Phys Rev 103 (1956) 511

9) W P Jesse and J Sadaukis, Phys Rev 107 (1957) 766

10) B Rossı, High Energy Partıcles (Prentıce-Hall, 1952) p 32

11) L Landau, J Exp Phys (USSR) 8 (1944) 201

12) K R Symon, Ph D Thesis (Harvard University, 1948) unpublished, a partial summary of this thesis is avalable ${ }^{10}$ ) 
13) P V Vavilov, Soviet Physıcs, JETP (Engl) 5 (1957) 749

14) S Seltzer and $M$ Berger, in Studies in Penetration of Charged Particles in Matter, NAS-NRC Publ no 1133 (1964) 187

15) G Igo and R M Eisberg, Rev Scı Instr 25 (1954) 450

16) J E Moyal, Phıl Mag 46 (1955) 263
17) See, for example, E D Cashwell and C J Everett, A practical manual on the Monte Carlo Method (Pergamon Press, 1959) p 9

18) O Blunck and S Leisegang, $Z$ Physık 128 (1950) 500.

19) O Blunck and K Westphal, Z Ph sik 130 (1951) 641. 